\title{
On the Design and Performance Evaluation of Peak to Average Power Ratio (PAPR) using Hybrid Transform Techniques in MIMO-OFDM
}

\author{
Javaid Ahmad \\ Shiekh \\ PG Department of \\ Electronics \& IT \\ University of \\ Kashmir \\ Hazratbal, Srinagar
}

\author{
Mehboob-ul- \\ Amin \\ PG Department of \\ Electronics \& IT \\ University of \\ Kashmir \\ Hazratbal, Srinagar
}

\author{
Sahir Majeed \\ PG Department of \\ Electronics \& IT \\ University of \\ Kashmir \\ Hazratbal, Srinagar
}

\author{
Sakeena Akhtar \\ PG Department of \\ Electronics \& IT \\ University of \\ Kashmir
}

\author{
Shabir Ahmad \\ Parah \\ PG Department of \\ Electronics \& IT \\ University of \\ Kashmir \\ Hazratbal, Srinagar
}

\begin{abstract}
In this paper various Hybrid techniques of keying and coding algorithm have been implemented to efficiently reduce the peak-to-average-power ratio (PAPR) problem that exists in MIMO-OFDM. A novel Selective Level Method (SLM) method is used as a basic technique which has been combined with other keying and transform techniques. Square root technique and Discrete Cosine Transform (DCT) technique have been used for the reduction of PAPR in case of coding algorithm. The combined techniques of square rooting and Discrete Cosine transform have been proposed for the reduction of PAPR in MIMO-OFDM system. Simulations results show that the significant reduction in PAPR as compared to the existing techniques used for the reduction of PAPR.
\end{abstract}

\section{Keywords}

Multiple Input Multiple Output (MIMO), Orthogonal Frequency Division Multiplexing (OFDM), Discreet Cosine Transform (DCT), Selective Level Mapping (SLM),

\section{INTRODUCTION}

The reason for high data rate obtained in LTE and LTEAdvanced is due to the incorporation of multi-antenna or MIMO (Multiple Input and Multiple Output) techniques. MIMO system employ multiple antennas both at transmitter and receiver and a definite correlation exists between transmit and receive antennas that forms an important aspect of the MIMO channel [1], [2]. MIMO is basically one of the forms of smart antenna technology consisting of the antenna arrays and possess smart signal processing algorithms. The algorithms are used to identify spatial signal signature such as the direction of arrival (DOA) of the signal, and use it to calculate beam-forming vectors, to track and locate the antenna on the mobile/target [3]. Orthogonal frequency division multiplexing (OFDM) can be used with a MultipleInput Multiple-Output (MIMO) to form one of the most eminent multi-carrier transmission schemes. The use of mu.3ltiple antennas at transmitter and receiver increases the diversity gain that results in enhancement of system capacity
[4]. The orthogonality in multiple sub-carriers leads to the efficient use of spectrum thereby reduces the bandwidth [5]. One of the bottleneck problems that exist in MIMO- OFDM wireless communication system is high Peak-Average Power Ratio (PAPR of OFDM signal), and various technique have been proposed to reduce it. The occurrences of high peaks in the transmitted OFDM signal gears the degradation of the system performance due to various non-linear effects like spectral spreading, intermodulation, and signal constellation that exist inherently in power amplifiers[6]. In this paper various hybrid techniques of keying and coding algorithms have been implemented and simulated. In case of keying algorithm technique the OFDM modulator has been implemented by Inverse Fast Fourier Transform (IFFT).The output of IFFT is given to the OSTBC encoder with variable number of transmit and receive antennas and various Multiple- ary route numbers are defined for both PSK as well as QAM modulation algorithm. A novel Selective level mapping method is used for calculating the peak-to-average power ratio for every route using empirical cumulative distribution function and the one with the minimum PAPR is selected for transmission. In case of coding algorithm techniques Discreet Cosine transform algorithm (DCT) and square root technique have been implemented for the reduction of PAPR .The proposed technique is the hybrid of both DCT and Square root. The results obtained have been plotted in terms of various graphs

\section{BACKGROUND STUDY}

In order to transmit a data from the mobile equipment to the network, a power amplifier is required to increase the strength of the signal to a level high enough to be picked by the network. The peak of the wave does not carry any significant information than the average power of the signal over time,thus, average power level of the signal is much more important in determining the transmission speed output of the signal.Thus lower the difference between the peak power and average power of the signal, lesser is the consumption of energy by power amplifier and longer will be the operating time of mobile device[7]. The PAPR of OFDM signal is defined as ratio between maximum power and the average power. Mathematically, PAPR is defined as 


$$
\operatorname{PAPR}\{s(t)\}=\max \left[\operatorname{Re}\left(s(\mathrm{t}) \mathrm{e}^{\mathrm{j} 2 \pi f_{\mathrm{c}} \mathrm{t}}\right]^{2} / E\left\{\left[\operatorname{Re}\left(s(t) e^{\left.j 2 \pi t f_{c}\right]^{2}}\right\}\right.\right.\right.
$$$$
=\max |s(t)|^{2} \mathbb{E}\left\{|s(t)|^{2}\right\}
$$

Where $\mathrm{s}(\mathrm{t})$ is the pass band signal.

In PSK/OFDM system with $\mathrm{N}$ sub-carriers, the maximum power occurs when all of the $\mathrm{N}$ sub-carrier components are added with identical phases. Assuming that $\mathrm{E}\{|\mathrm{s}(\mathrm{t})|\}^{\wedge} 2=1$, it results in PAPR $=\mathrm{N}$, that is maximum power is equivalent to $n$ times the average power. As Per ITU standards, we have $\mathrm{N}=64$ used subcarriers. The maximum expected PAPR is thus $64(\sim 17.3 \mathrm{db})$.In this paper, a hybrid technique of DCT and Square root have been proposed to significantly reduce the PAPR below 17.3db.A brief overview of literature consulted during the work is as follows:

1. Wisam F.Al-Azzo et-al [8] proposed "Adaptive SquareRooting Companding techniques for PAPR reduction in OFDM system". The proposed technique changes the statistical characteristics of the OFDM signal to Gaussian distribution. The shifting of the characteristics from the statistical distribution to Gaussian distribution reduces the PAPR of the OFDM signal up to $6 \mathrm{db}$ for 64 QAM modulation scheme.

2. J.A Sheikh et-al [6] proposed "PAPR reduction in MIMOOFDM System using the Combination of OSTBC Encoder and Spreading code Sequence". In this paper the authors suggested the reduction in PAPR with the increasing value of M-Ary route number for each antenna configuration. On changing the number of transmitting antennas to 3 , there is a $3.75 \mathrm{db}$ reduction in PAPR for 16 PSK modulation scheme.

3. Montadar Abas et-al [9] proposed "Reducing the PAPR of OFDM Systems by Random Variable Transformation" In this paper a side information free technique based on the concept of random variable transformation has been proposed in . The suggested method transform, Random variables (RV'S) into other RV'S aiming to reshape the constellation that will consequently produce OFDM symbols with a reduced peak to average power ratio. The PAPR of the Frequency domain RFD-RVT is reduced to around $1 \mathrm{~dB}$ for both $16 \mathrm{PSK}$ and 16 QAM modulations mapping, and the PAPR of TD-CR is reduced by $2.25 \mathrm{~dB}$ for the 16-PSK modulation mapping and by $1 \mathrm{~dB}$ for the 16-QAM mapping.

4. Syed Hassan Ahmed et-al [10] proposed "Performance evaluation of DVB-T based OFDM over wireless communication channels". In this paper DVB-T (international based digital video broadcasting) based OFDM is analyzed in terms of various parameters over different communication channels. DVB-T $2 \mathrm{k}$ and $8 \mathrm{k}$ mode carriers are transmitted over AWGN Rican and Rayleigh channel, the received signal is thus corrupted. The corrupted signal is thus manipulated in terms of different values of SNR (ranging from 0db to $16 \mathrm{db}$ ) and modulation schemes (4 QAM, 16QAM and 64 QAM).

5. Suverna Sengar et-al [11] proposed "Performance improvement in OFDM system by PAPR reduction". In this paper two different PAPR reduction methods, partial transmit sequence (PTS) and selective mapping (SLM) are used for the reduction of PAPR. With 64 sub-carriers chosen, it can be seen original signal is having a PAPR of $11 \mathrm{db}$.The SLM method reduces the PAPR to $8.8 \mathrm{db}$ and PTS method reduces the PAPR to about $7.1 \mathrm{db}$. For 128 sub-carriers the original signal is having a PAPR of $10.2 \mathrm{db}$. The SLM method reduces the PAPR to $8.2 \mathrm{db}$ and PTS method reduces the PAPR to about $6.4 \mathrm{db}$.

\section{PROPOSED METHODOLOGY}

The proposed work is based on the Monte- Carlo simulation study carried out on MIMO-OFDM system using keying and coding. In case of keying algorithm technique 64 sub-carriers are chosen and oversampling factor of 8 is used. Various PSK constellation and QAM constellation symbols are selected. OFDM symbols are generated in the frequency domain as an array of 0 's and 1's.The IFFT of generated signal is passed through the OSTBC Encoder having variable number of transmit antennas and different $\mathrm{M}$-ary phase number are defined $(\mathrm{M}=2,4,8,16$ in case of PSK constellation and $\mathrm{M}=16,32,64,128,256$ in case of QAM constellation symbols). A novel SLM algorithm is applied and the signal's corresponding PAPR empirical cumulative distribution function (ECDF) is calculated for each M-ary route number. The signal's complementary cumulative distribution function (CCDF) for different PAPR values is plotted in terms of graphs

In case of coding algorithm DCT and SQRT technique are applied to reduce the PAPR. The SQRT technique provides sufficient reduction in peak, hence maintaining good autocorrelation. DCT transform spreads the signal, thereby reducing the peak. The hybrid technique i.e. the combination of two techniques that is SQRT and DCT further helps in the reduction of PAPR and gives us the best possible result

\section{RESULTS AND DISCUSSIONS}

Case 1. PAPR reduction for keying algorithm technique.

In this case Sixty four carriers have been used and oversampling factor is eight. The specifications in this work have been made as per International Telecommunication Union (ITU). The graphs are plotted between CCDF and $\mathrm{PAPR}_{0}(\mathrm{db})$. The simulation result for PSK constellation symbols using conventional SLM technique has been shown in figure 1. With CCDF (Pr $\left[\mathrm{PAPR}>\mathrm{PAPR}_{0}\right]$ ) equal to $\max$ (i.e. 1), it can be shown that PAPR decreases with increasing values of $M$.For $M=16$ PAPR reduces to $7 \mathrm{db}$ as compared to original signal of $10.75 \mathrm{db}$, thus there is a reduction of $3.75 \mathrm{db}$, for $\mathrm{M}=2$ PAPR reduces to $9.2 \mathrm{db}$, for $\mathrm{M}=4$ PAPR reduces to $8 \mathrm{db}$, and for $\mathrm{M}=8$ PAPR reduces to $7.6 \mathrm{db}$.

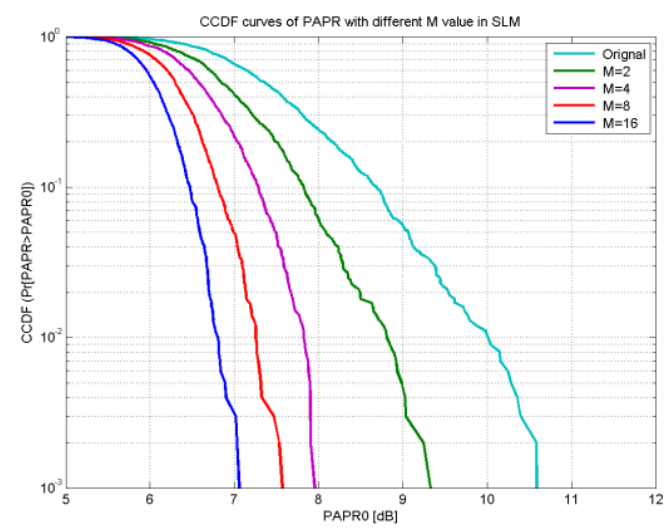

Fig 1.Plot for PSK constellations using conventional SLM

Figure 2 shows the CCDF curves of PAPR for different QAM modulations. Again it can be seen that PAPR reduces with the increasing values of $\mathrm{M}$. For $\mathrm{M}=256 \mathrm{PAPR}$ is minimum. The original signal is having a PAPR of $11.9 \mathrm{db}$. For M=16 PAPR reduces to $7 \mathrm{db}$, for $\mathrm{M}=32 \mathrm{PAPR}$ reduces to $6.7 \mathrm{db}$, for $\mathrm{M}=64$ 
PAPR reduces to $6.6 \mathrm{db}$, for $\mathrm{M}=128$ PAPR reduces to $6.1 \mathrm{db}$ and for $\mathrm{M}=256 \mathrm{PAPR}$ reduces to $5.9 \mathrm{db}$.

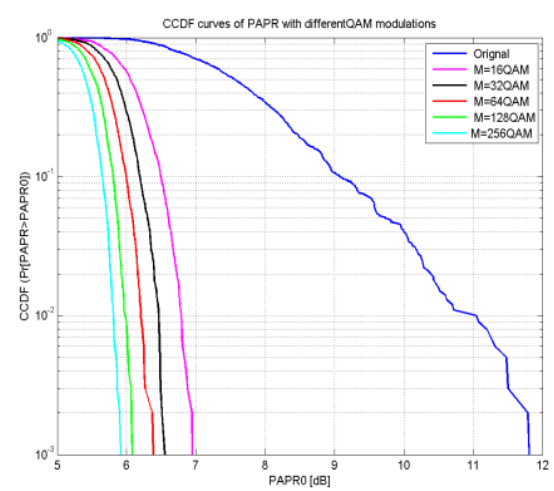

Fig 2. Plot for QAM constellations using conventional SLM

Case 2. PAPR reduction using various coding techniques.

In this case DVB-2k based MIMO-OFDM signal is generated using DVB parameters. The specifications used in our work are shown in Table 1.

Table 1. Parameters of Simulation

\begin{tabular}{|l|l|}
\hline $\begin{array}{l}\text { Useful OFDM symbol } \\
\text { period ,Tu }\end{array}$ & $224 \mathrm{e}-6$ \\
\hline $\begin{array}{l}\text { Baseband elementary period } \\
\mathrm{T}\end{array}$ & $\mathrm{Tu} / 2048$ \\
\hline Allowed Guard Interval G & $1 / 4,1 / 8,1 / 16$, and $1 / 32$ \\
\hline Guard band duration delta & $\mathrm{G} * \mathrm{Tu}$ \\
\hline Total OFDM symbol period & $\mathrm{Ts}=\mathrm{delta}+\mathrm{Tu}$ \\
\hline Number of subcarriers Kmax & 1705 \\
\hline Kmin & 0 \\
\hline IFFT/FFT length, FS & 4096 \\
\hline $\begin{array}{l}\text { Carrier period to elementary } \\
\text { period ratio q }\end{array}$ & 10 \\
\hline carrier frequency, fc & $\mathrm{q} * 1 / \mathrm{T}$ \\
\hline
\end{tabular}

The MIMO-OFDM signal generated after using the parameters shown in the table1 is passed through the OSTBC Encoder The encoder encodes the information symbols from the QAM Modulator by using complex orthogonal codes for two, three, and four transmit antennas. The number of transmit antenna is given to this block as an input. The basic idea to use DCT transform in MIMO-OFDM is to reduce the autocorrelation of the input sequence to reduce the peak to average power problem and it requires no side information to be transmitted to the receiver. The out-put of the OSTBC Encoder is combined with DCT matrix. The DCT matrix spreads the signal thereby reducing the peak. The proposed hybrid technique i.e., the combination of DCT and Square root reduces the PAPR significantly. The probability set is chosen between 0 and 100 . Figure 3 shows the simulation result when number of transmitting antennas are changed to 2 .

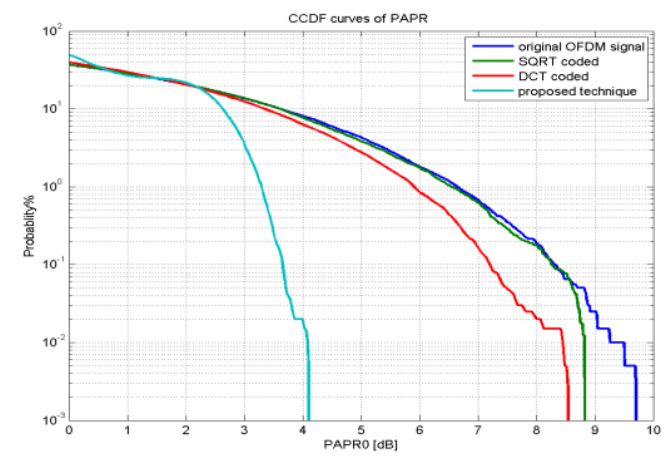

Fig 3. Plot for proposed techniques with num $\mathrm{Tx}=2$

The original signal is having a PAPR of $9.8 \mathrm{db}$, with the implementation of square root technique the PAPR reduces to $8.9 \mathrm{db}$.The DCT transform reduces the PAPR to 8.77 , and the proposed technique i.e., $\mathrm{DCT}+\mathrm{SQRT}$ reduces the PAPR to $4.1 \mathrm{db}$, thus there is the reduction of $5.9 \mathrm{db}$.

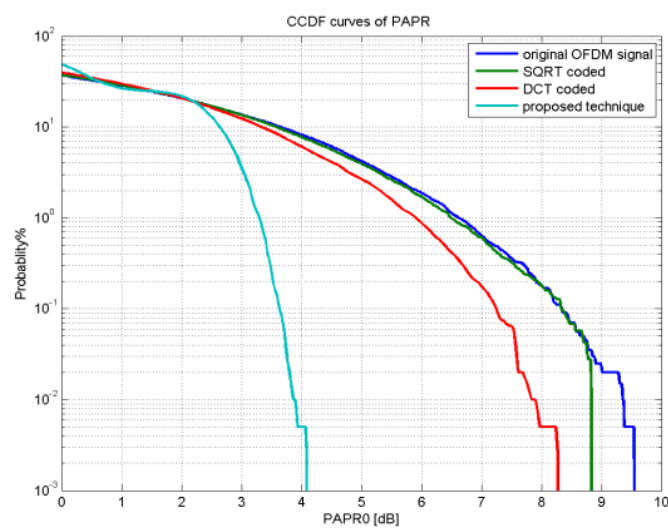

Fig 4. Plot for proposed techniques with numTx=3

Figure 4 shows the simulation result when number of transmit antennas are changed to 3 . The original signal is having a PAPR of $9.7 \mathrm{db}$, SQRT technique reduces the PAPR to $8.9 \mathrm{db}$.DCT technique reduces the PAPR to $8.15 \mathrm{db}$ and the proposed technique reduces the PAPR to $4 \mathrm{db}$, thus there is a reduction of $5.7 \mathrm{db}$ as compared to original signal.

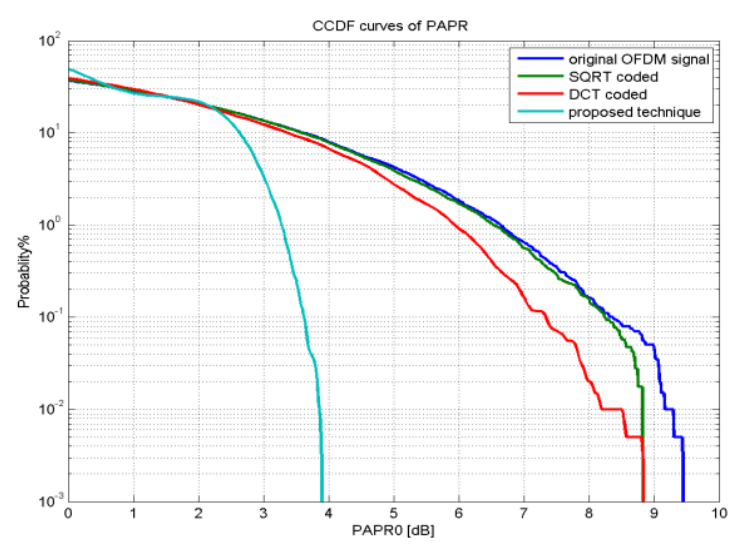

Fig 5. Plot for proposed techniques with numTx=4 
Figure 5 shows the simulation result when number of transmit antennas are changed to 4.The original signal is having a PAPR of $9.5 \mathrm{db}$, SQRT technique and DCT technique reduces the PAPR to $8.9 \mathrm{db}$ and the proposed technique reduces the PAPR to $3.9 \mathrm{db}$, thus there is a reduction of $5.6 \mathrm{db}$ as compared to original signal.

\section{CONCLUSION}

The above mentioned analysis represents a conservative estimate of PAPR reduction in MIMO- OFDM systems using hybrid techniques. The PAPR is one of the major drawbacks of multi-carrier communications. Various techniques have been proposed to reduce the PAPR in multi-carrier communication systems. From the simulation results it has been observed that hybrid techniques of square root and discrete cosine transform outperforms the other conventional techniques. The proposed techniques have a lot of scope in 4G LTE communications particularly in downlink.

\section{REFERENCES}

[1] Dahlman, E., Parkvall, S. and Sköld, J. (2011) 4G LTE/LTE-Advanced for Mobile Broadband, Elsevier.

[2] Browne, M. and Fitz, M. (2006) Singular value decomposition of correlated MIMO channels. IEEE Global Telecommunications Conference (GLOBECOM) 2006.

[3] Weima,Sen,zhehu,YancongWang,Liangzhue (2009) “ Cooperative Spectrum Sensing in OFDM on MIMO Cognitive Radio sensor networks" IEEE Wireless communications ,Networking and Mobile computing, Wicom 2009

[4] Anand S. Konanur, Keyoor Gosalia,Sandeep H. Krishnamurthy Brian Hughes, Gianluca Lazzi,(2005) "Increasing Wireless Channel Capacity Through MIMO Systems Employing Co-Located Antenna" IEEE Transactions on microwave theory and techniques, vol. 53, no. 6, June 2005 pp-1837
[5] KingF.leu, DouglasB.Williams (2004) "Bandwidth efficient OFDM transmitter diversity techniques".EURASIP journal on Applied signal processing Vol 1. 2004 pp 1508-1519

[6] Javaid A. Sheikh, Shabir A. Parrah, G. Mohiuddin Bhat ,Mehboob ul Amin (2013) "PAPR reduction in MIMOOFDM system using combination of OSTBC encoder and Spreading Code Sequences", Proceedings of IEEE International Conference on Multimedia Signal Processing and Communication Technologies- IMPACT (AMU, Aligargh-23- 25 Nov.) pp 11-15.

[7] Mehboob ul Amin, Javaid Skeikh, S.A Parrah, G.M Bhat, "A new method for PAPR reduction in OFDM using Embedded Transform Techniques", Elixir Network Engg ISSN 2229-712X(In press)

[8] Wisam F. Al-Azzo and Borhanuddin Mohd. Ali (2011) "Adaptive Square-Rooting Companding Technique for PAPR Reduction in OFDM Systems" World Academy of Science, Engineering and Technology 75

[9] Montadar Abas Taher, Mandeep Jit Singh, Mahamod Bin Ismail, Salina Abdul Samad, and Mohammad Tariqul Islam (2013) "Reducing the PAPR of OFDM Systems by Random Variable Transformation" ETRI Journal, Volume 35, Number 4, August

[10] Syed Hassan Ahmed, Syed Muhammad Umar Talha, Aamir Khan, (2012) "Performance evaluation of DVB-T based OFDM over wireless communication channels," Proceedings of the international multi conference of engineers and computer scientists 2012 vol1,IMECS,march 14-16, Hong Kong

[11] Suverna Sengar, Partha Pratim Bhattacharya, (2012 )"Performance improvement in OFDM System by PAPR reduction” Signal \& Image Processing : An International Journal (SIPIJ) Vol.3, No.2, April 